\title{
The Past, Current and Future Colour of Outdoor Lighting of Japan- Standing out from the Rest of the World
}

\section{Spiros KITSINELIS}

www.the-nightlab.com

Received June 14, 2012, Accepted September 27, 2012

Looking at the photos of cities at night taken by NOAA's DMSP satellite, one can only be overwhelmed by the accidental beauty of the lit urban areas that signify the human presence and progress ${ }^{1)}$. Indeed in the eyes of an observer in space, the light from cities at night is the only evidence of our existence and the lighting technologies are the only visible human products. A global picture of these lights, as composed by the satellite reveals the most developed places on the planet in terms of economic and industrial activity.

It is also true that light reaching the skies signifies waste and a kind of pollution that affects the lives of all living things so the pressure is on by various groups to minimise this waste for both economic and environmental reasons. Perhaps in future decades this will be achieved and light will be directed towards our activities and living spaces but not upwards. But what another fascinating thought is triggered by this about the time we live in. A couple of hundred years ago and a couple of hundred years from now the planet did not and will not look like that at night.

However this image that most people are familiar with is panchromatic so individual colours (and lighting technologies) cannot be distinguished.
Now, thanks to photographs taken by the astronauts of the International Space Station ${ }^{23)}$ over the past decade we can see the lit cities at night in colour. The colour they emit is due to the dominant lighting technology that is employed by the city for outdoor lighting.

The technologies have certainly changed over time. From burning oil and gases we moved to the incandescent lamp when electricity penetrated our lives. The next technology that was adopted for outdoor lighting was the one based on mercury (low pressure fluorescent in the $30 \mathrm{~s}$ and high pressure in the $50 \mathrm{~s}$ ). But the light sources that dominated outdoor lighting due to their record high efficacy values were the sodium vapour discharge lamps. The low pressure version reaches $200 \mathrm{~lm} / \mathrm{W}$ as its monochromatic yellow emission at $589 \mathrm{~nm}$ is near the peak of the human eye photopic sensitivity curve ${ }^{45)}$ and high pressure versions can reach more than a $100 \mathrm{~lm} / \mathrm{W}$. On the other hand most mercury vapour lamps reach values well below $100 \mathrm{~lm} / \mathrm{W}$.

A look at all these photos ${ }^{233}$ from space verifies the known fact that sodium is the undisputed king of outdoor lighting and that the light source technologies based on this element dominate the adorned face of the

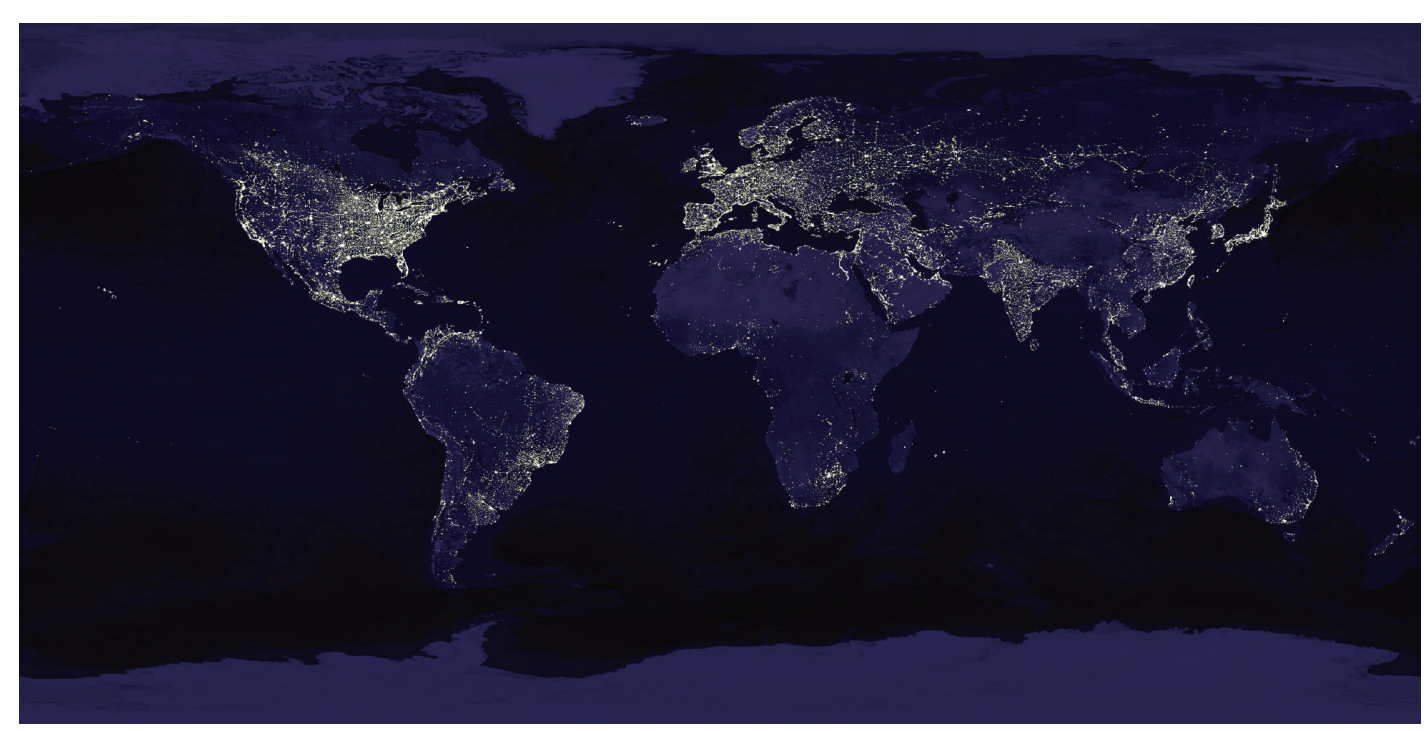

Figure 1 Mapping of the night sky by the DMSP satellite (October 2000 data) 


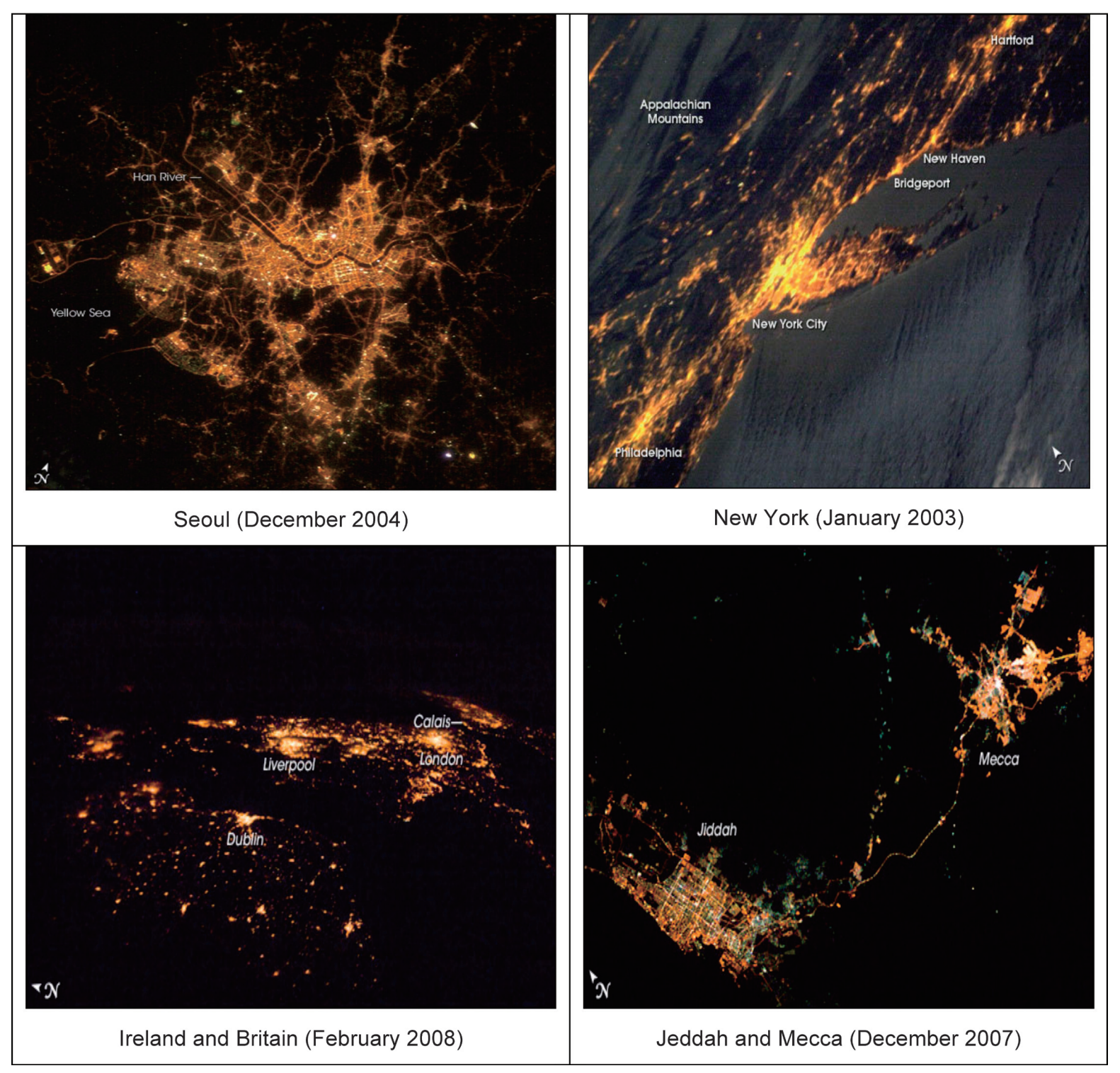

Figure 2 Major cities photos from space

earth at night and of every major city...except one.

The exception to this as revealed by the astronauts' photos is the mega city of Tokyo, capital of Japan and considered by many as the largest city on earth. This metropolis may be the Empress of all cities but does not honour the king of all outdoor lamps as sodium doesn't seem to be the ornament of choice. In contrast to the yellow/orange glow that dominates all other photos, Tokyo appears with a greenish glow that is probably due to the use of mercury based lamps (and a portion of metal halide lamps). It is known after all that one of mercury's strongest emission lines in the visible is the green line at $546.1 \mathrm{~nm}$. And if sodium lamps are not used extensively this leaves with mercury and its many lamp versions to consider assuming that the lighting infrastructure is the same for the last few decades and no new technologies have been adopted to such an extent yet.
The Japanese are known for their pioneering work and their early adoption of new technologies. Their train transportation infrastructure for example that seems so futuristic and advanced to the eyes of most people, has in fact been developed more than half a century ago as part of a makeover and an update with view to the Tokyo Olympic games of 1964 .

So the Japanese probably adopted the latest technology of the time to illuminate their roads and other outdoor spaces but retained the same technology with no changes or updates since then. The answer to why the Japanese did not adopt sodium lighting in the decades that followed, can give important information on the lighting culture and market possibilities in this country. Sodium discharges would cause less light pollution (less scattering for longer wavelengths) and save energy (due to the higher efficacies) but it seems that these are not the most important criteria for the Japanese when 


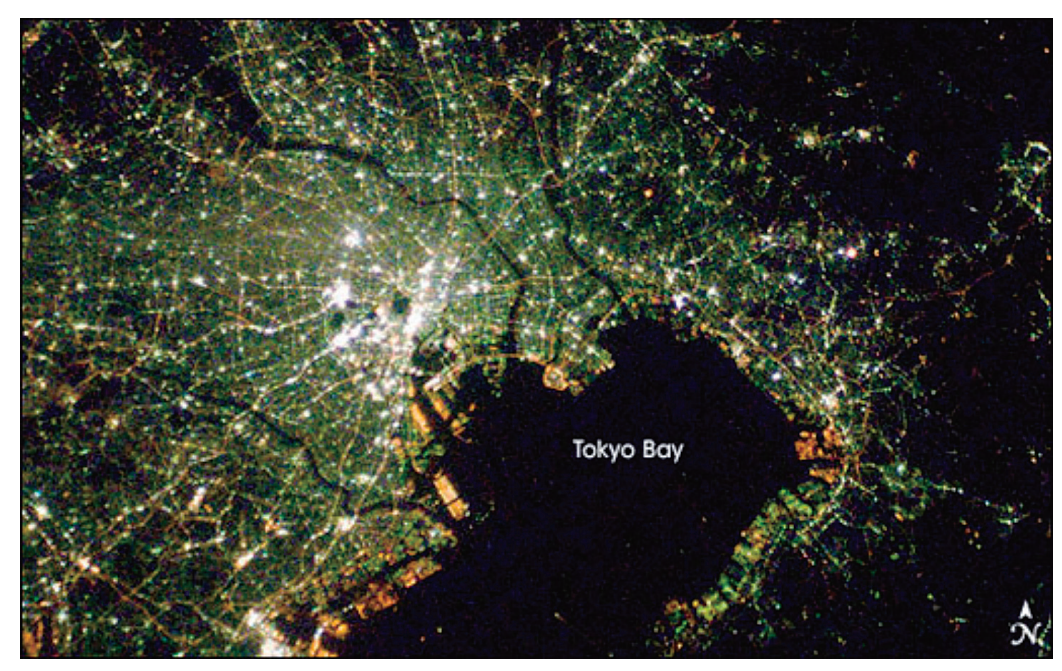

Figure 3 Tokyo (February 2008)

choosing lighting technologies.

An obvious conclusion is that there is a preference for sources with good rendering properties (better than those of most sodium lamps). Another would be that they prefer sources with higher colour temperatures and cool white light. A third could be that they prefer their lighting environments to be stable so they avoid the use of different light source technologies.

Today there are good arguments for the use of metal halide $(\mathrm{MH})$ or LED systems for outdoor spaces with one of them being the need for bluish emissions (cool white) that aid peripheral and mesopic vision and another being the sturdiness and long lifetime of solid state technologies. In fact LEDs have found widespread use already in some applications such as traffic lights ${ }^{3 / 4)}$.

These technologies can offer the choice of colour temperature and good colour rendering properties. The future looks bright and time will tell the colour of Tokyo but I wouldn't expect it to get any warmer. If sodium lamps did not penetrate the Japanese market for outdoor lighting until now, probably they never will and the greenish glow of Japanese cities, if changed, will probably be replaced by the cool (bluish) or neutral white colour of metal halides and/or LEDs.

\section{References}

(1) Defense Meteorological Satellite Program (DMSP) http://www.ngdc.noaa.gov/dmsp/ http://earthobservatory.nasa.gov/IOTD/view.php?id= 896]

(2) Cities at night: The view from space http://earthobservatory.nasa.gov/Features/ CitiesAtNight/

(3) The gateway to astronaut photography of Earth http://eol.jsc.nasa.gov/

(4) Kitsinelis, S.: Light Sources: Technologies and Applications, Taylor and Francis, 2010 ISBN 9781439820797

(5) Kitsinelis, S.: The Right Light: Matching Technologies to Needs and Applications, Taylor and Francis, 2012, ISBN 978-1439899311 